\title{
On Dynamical Situations in Vehicle Routing Problems (DSVRP)
}

\author{
Felix Makanjuola Aderibigbe, Kayode James Adebayo* \\ Department of Mathematics, Faculty of Science, Ekiti State University, Ado Ekiti, Nigeria \\ Email address: \\ felixaderibigbe@eksu.edu.ng (F. M. Aderibigbe), kayode.adeabyo@eksu.edu.ng (K. J. Adebayo) \\ ${ }^{*}$ Corresponding author
}

\section{To cite this article:}

Felix Makanjuola Aderibigbe, Kayode James Adebayo. On Dynamical Situations in Vehicle Routing Problems (DSVRP). American Journal of Traffic and Transportation Engineering. Vol. 6, No. 1, 2021, pp. 1-9. doi: 10.11648/j.ajtte.20210601.11

Received: January 12, 2020; Accepted: January 21, 2020; Published: January 12, 2021

\begin{abstract}
This paper discusses the formulation of a stochastic Vehicle Routing Problem (VRP) process, introduced to existing deterministic VRP process, directing all efforts towards the dynamical situations that occur in a typical VRP setting as it applies to everyday real-life situations. It contrasts the dichotomy between static and dynamical situations in VRP, constructs and analyzes the relationship between static and dynamical situations in Vehicle Routing Problems, formulates an expression to describe the dynamical situation in VRP and relates the problem formulation to existing VRP with a view to solving the problem as it applies to real-life situations, a major trend in the business world today. With a view to achieving these, this paper associates the merits of reactivation of route, re-optimization of operation process and projects an anticipatory demand for a dynamical situation in VRP with stochastic requests via three stages: Pre-Decision States, Decisions States and Post-Decision States. First, as a reaction to anticipatory customers' requests, the current routing plans need to be re-optimized and current customers' request reactivated. Second, potential future requests need to be anticipated along current decision making since life itself is dynamic. Decisions need to be made in good time. Though, the limited time frame between when a vehicle leaves and returns to the depot often prohibits extensive optimization in both dimensions rather, answer the questions that arise on how to utilize the limited time effectively and judiciously, satisfying both the current and anticipatory customers equally.
\end{abstract}

Keywords: Dynamical Situation, Static Situation, Vehicle Routing Problem, Re-optimization, Anticipatory Routing, Stochastic Demand, Pre-Decision State, Post-Decision State

\section{Introduction}

Vehicle Routing Problems (VRP) have attracted attention in the last three decades due to the increased interest in various geographical solutions and technologies the use of the model in solving logistics and transportation issues. Many researchers have used heuristic approaches to solve Vehicle Routing Problems where specified situations and constraints are explicitly analyzed. The uncertainties that go along with real-life situations that make life dynamical is an extension of the VRP to which action will be focused. Solving this kind of problem is quite challenging. It requires optimization along different directions and dimensions.

Literatures on VRP over years have become very robust, encompassing and covering a variety of applications, real life modelling approaches and solution methods. According to Jean-Francois, C., et al., (2007), it was remarked that, VRP lies in the heart of distribution management [1]. It is being faced each day by thousands of companies and organizations engaged in the delivery and collection of goods, people or services. Due to varied conditions from one setting or the other, the objectives and constraints encountered in practice vary widely. Indeed, these problems have caught the attention of many researchers and motivated a broad range of collaborations between companies and the academia because of its importance to making life worth living.

As widely acclaimed, the VRP is a generalization of the Travelling Salesman's Problems (TSP) in the sense that, the VRP consists of determining $m$ vehicle routes, where a route is a tour that begins at the depot, connects a subset of the customers in a given order and returns to the depot. All the customers must be visited exactly once and the entire quantity of goods that customers demand on a route must not exceed the carrying capacity of the vehicle. The aim of the 
VRP is to reduce the cost of distribution.

In real-life distribution context, Christofides, N., (1976), noted that, a number of constraints complicate the model [2]. The side constraints could be time constraints on the total route time, time windows within which the service must be completed, heterogenous fleet and split delivery. In addition, vehicle routing problems lead to formulations that are challenging and tasking. This requires the development of sophisticated solution strategies and motivate the design of clever heuristics and meta heuristics.

It is opined that, the VRP is a generic name given to a whole class of problems involving the visit to several customers by vehicles [3]. The VRP has been named variously: vehicle scheduling by Zaepfel, G. and Boegl, M., (2008), vehicle dispatching by Du, T., Wang, F. K., and Lu, P. Y., (2007), or simply as the delivery problem by Gendreau, M., Guertin, F., Potvin, J. Y., and Seguin, R., (2006) [4-6]. Take for example, the pickup of children by school buses, house-call tours by a doctor, the collection of mails from mail boxes, the delivery of laundry, preventive maintenance inspection tours, etc. These are all VRPs in which the delivery operation is a collection, collection and delivery, or delivery alone; in which the goods and vehicles can take a variety of forms, some of which may be physical in nature.

The VRPs have been studied with much interest within the last three to four decades [7]. The majority of works focus on the static and deterministic cases of vehicle routing such that all information is known at or before the time of planning of the routes. In most real-life applications, though, stochastic and/or dynamic information occurs parallel to the routes being carried out.

Real-life examples of stochastic and/or dynamic routing problems include the distribution of oil to private households, the pick-up of courier mail/packages and the dispatching of buses for the transportation of elderly and handicapped people. In these examples the customers' profiles (i.e. the time to begin service, the geographic location, the actual quantity demanded etc.) may not be known at the time of the planning or even when service has begun for the advance request customers.

Two distinct features make the planning of high-quality routes in this environment much more difficult than in its deterministic counterpart; firstly, the change that may occur after setting out of the vehicle and secondly, is the time horizon within which the process must be completed.

\section{Uncertainty of Real-Life Situations}

An extension of the VRP to the real-life application is basically a Dynamical situation. This takes into consideration the uncertainties of life which could result from the customers or the vehicle route planner. For instance, in some applications, there may be uncertainty about customers which are initially unknown but revealed incidentally while the operations are ongoing. There can also be uncertainties in the route planning. For example, when the locations and/or the demands of customers are unknown at the beginning but revealed over time after the vehicles have already been dispatched.

Applications of the Dynamical situation in VRP include real-time ambulance relocation problem, real time vehicledispatching system for consolidating milk runs, real-time goods delivery to customers, and local area courier services [8-11]. Since the problem is dynamical, it is required to adaptively modify the existing solution pattern in order to take care of the newly revealed customers.

The literature reviewed identified stochastic customer's requests as the main drive for uncertainty in practice these days [12]. Hence, the following were identified: emergency vehicles, technical and healthcare dispatch, courier services as well as parcel and passenger transportation in the growing fields of same-day delivery, shared mobility, or demand responsive transportation. Other examples of uncertainty are stochastic service times, stochastic demands and travel times discussed [13-15].

While dynamical routing applications differ in their objectives and constraints with uncertain requests, they all share the requirement of assigning new requests to vehicles and of determining efficient routing plans for the fleet except that routing is done ahead of dispatch.

On the operational level, both are limited in their resource's accessibility such as number of vehicles, working hours of drivers and carrying capacity of vehicles. Most of the approach aim at utilizing these limited resources to maximize revenue or the number of served customers. Because drivers and customers alike often expect fast responses, hence, decision making needs to be taken in realtime and the allotted time for the computational calculations found to be highly limited [16].

The most efficient way of doing this is to modify a part of the solution process and inject the new customers into the existing solution line. To achieve a better plan, it is wise to take future customers into account else the business downs, customers are lost to other competitors or are not satisfied. A double-horizon heuristic for solving a dynamic pickup and delivery problem, which focuses on both a short-term goal and a long-term goal [17]. While the former minimizes the total distance travelled, the latter maximizes the slack time to ease the insertion of future customers. Several authors have investigated the waiting strategies to be employed and improved the solution by letting vehicles wait at certain places for proper timing to mention a few authors are [17-19].

\section{Dichotomy Between Static and Dynamical Situations in VRP}

As it will be demonstrated throughout this paper, the time when relevant information is made known to the planner distinguishes a dynamical situation in Vehicle Routing Problem from a static situation. At this point, to guide toward solving this class of VRP, it is necessary to dichotomize between a static and dynamical situation in VRP. The following criterion can be used to classify static routing 
problem: "if the output of a certain formulation is a set of pre-planned routes that are not re-optimized and are computed from inputs that do not evolve in real-time". On the other hand, "refer to a problem as dynamic if the output is such that, it is not a set of routes, rather a policy that prescribes how the routes should evolve as a function of those inputs that have not evolve especially in real-time". In the above, the temporal dimension plays a vital role for categorizing vehicle routing problems.

Definition 1.1: Static Situation

A situation in VRP is said to be static if all information relevant to the planning of the routes a priori is assumed to be known by the planner before the routing process begins and such information relevant to the routing process do not in any way change after the routes have been constructed. The information assumed to be relevant include: all attributes of the customers, such as the geographical location of the customers, the on-site service time and the quantity demanded by the customer. Furthermore, system information such as, the travel times of the vehicle between two successive customers must have been known by the planner from the onset.

Definition 1.2: Dynamical Situation

A VRP in which not all the information relevant to the planning of the routes is known by the planner as at the time the routing process begins is called a dynamical situation. In such a case, the information earlier gathered can change after the initial routes have been constructed hence, bringing about routing modifications, an alteration that could come in form of addition or subtraction as it affects the attributes of the customer and the system information.

The dynamical situation in VRP calls for online algorithms that work in real time since the immediate requests should be served, if possible. It is not always easy to find solutions to problems of realistic sizes in a reasonable amount of computation time. This means that, for the dynamical situation in VRP, a class of NP-hard problems must be solved each time a new request is received. The solution of the current problem was referred to as a tentative solution [20]. The tentative solution therefore corresponds to the current set of inputs and only those that have placed requests earlier. Should there be no new requests received during the execution of this solution, the tentative route becomes the optimal route.

\section{Problem Statement}

In practice, the Dynamical Situations in Vehicle Routing Problems (DSVRP) objective function can be quite complex to state. It may include the minimization of the difference between the longest and shortest routes, balance of workload among drivers, the minimization of number of vehicles to reduce overhead costs, and/or the maximization of the number of serviced customers to improve the service level.

In some applications, robustness of the solution is also one of the important objectives. However, these different objectives may conflict with one another. In here, we refer to the initially known customers as Early Request Customers (ERC),

$$
E R C=\sum_{i=1}^{N} C_{i}
$$

where $N$ is the number of customers. The stochastically requesting customers, Late Request Customers (LRC) is given as:

$$
L R C=\sum_{i=1}^{x} C_{N_{S}}^{i}
$$

where $x$ is the number of anticipatory customers, and $N_{S}$ represents the number of customers which have been serviced as at the time the LRC was to be serviced.

A vehicle serves customers in a service area within a time horizon. The tour starts and ends at the depot with a set of ERC that are known before the tour. The ERC is compulsory to be served. In the course of the tour, a new set of LRC may stochastically request service.

The LRCs remain unknown until their requests are placed. Their request times and locations follow a known probability distribution. Whenever the vehicle services a customer, the dispatcher decides on (i) the subset of occurred requests to be assigned to the vehicle, (ii) an update of the planned tour as long as the vehicle is still within the service period. If an LRC is assigned, the vehicle is required to serve this customer in the remainder of the time horizon. The dispatcher aims at maximizing the number of assigned LRC subject to the degree of dynamism.

\subsection{The Dynamical Degree (DD)}

Dynamical Situations in Vehicle Routing Problems (DSVRP) with stochastic requests differ in their level of uncertainty. In particular, they differ in the question of how many customers are initially known and how many customers may stochastically make requests over the time horizon.

The expected uncertain requests percentage is referred to as the Dynamical Degree or Degree of Dynamism (DD) [21]. This is defined as the ratio of expected numbers of LRC to Overall Customers (OC):

$$
D D=\frac{\mathrm{LRC}}{\mathrm{OC}}
$$

The Overall Customers $(O C)$, the total number of ERC and LRC to be considered have three probable ways of occurrence as:

In the first kind, the LRC comes after all the ERC orders have been met. From (4.1) and (4.2) the

$$
\mathrm{OC}=\mathrm{ERC}+\mathrm{LRC}=\sum_{i=1}^{N} C_{i}+\sum_{i=1}^{x} C_{N_{S}}^{i}
$$

where $N$ is number of ERC customers and $x$ is the number of LRC customers and $N_{S}$ is the number of customers in LRC that have been attended to. In this case, the ERC initially planned tour is not affected in any way rather, it gives room for anticipatory demand planned alongside the tour to be attended to.

The $D D$ from (3) and (4) in this case is given by: 


$$
\mathrm{DD}=\frac{\mathrm{LRC}}{\mathrm{OC}}=\frac{\mathrm{LRC}}{\mathrm{ERC}+\mathrm{LRC}}=\frac{\sum_{i=1}^{x} C_{N}^{i}}{\sum_{i=1}^{N} C_{i}+\sum_{i=1}^{x} C_{N_{S}}^{i}}
$$

Second, is a case whereby the $L R C$ comes after a fractional part of the $E R C, E R C(1)$, orders have been met and the remaining $E R C$ ie $E R C(2)=E R C-E R C(1)$, orders are met after all possible LRC have been serviced thus:

$$
\mathrm{OC}=\mathrm{ERC}(1)+\mathrm{LRC}+\operatorname{ERC}(2)
$$

where $E R C(1)+E R C(2)=E R C$ and the resulting $O C$ in the second case is thus:

$$
O C=\sum_{i=1}^{G_{1}} C_{i}+\sum_{i=1}^{x} C_{N_{S}}^{i}+\sum_{i=G_{1}+1}^{N-G_{1}} C_{i}
$$

where $G_{1}<N$, represents the customer in turn in the ERC that has just been serviced after that the LRC demand is to take effect and $x \leq k$ represents the maximum possible LRC that can be taken into consideration such that the initial VRP plan will not be uttered or affected.

Also, from (3) and (7), the DD in the second case is given by:

$$
\begin{aligned}
& \mathrm{DD}=\frac{\mathrm{LRC}}{\mathrm{OC}}=\frac{\mathrm{LRC}}{\operatorname{ERC}(1)+\mathrm{LRC}+\operatorname{ERC}(2)} \\
& D D=\frac{\sum_{i=1}^{x} C_{N_{S}}^{i}}{\sum_{i=1}^{G_{1}} C_{i}+\sum_{i=1}^{x} C_{N_{S}}^{i}+\sum_{i=G_{1}+1}^{N-G_{1}} C_{i}}
\end{aligned}
$$

The third case is a generalized situation whereby the LRC comes intermittently within the ERC except that, it can't come before the first ERC thus:

$$
L R C=\sum_{i=1}^{x_{1}} C_{G_{1}}^{i}+\sum_{i=1}^{x_{2}} C_{G_{2}}^{i}+\cdots+\sum_{i=1}^{x_{n-1}} C_{G_{n-1}}^{i}+\sum_{i=1}^{x_{n}} C_{G_{n}}^{i}
$$

where $x_{1}, x_{2}, \ldots, x_{n-1}, x_{n}$ represent various number of anticipatory customers coming up intermittently and the corresponding $O C$ is given by:

$$
O C=E R C(1)+L R C(1)+E R C(2)+L R C(2)+\cdots+L R C(y)+E R C(M)
$$

where $M \leq N$ and $y \leq x$. Also,

$$
E R C(1)+\cdots+E R C(M)=E R C
$$

and $\operatorname{LRC}(1)+\cdots+\operatorname{LRC}(y)=L R C$.

The resulting $O C$ in the third case is thus:

$O C=\sum_{i=1}^{G_{1}} C_{i}+\sum_{i=1}^{x_{1}} C_{G_{1}}^{i}+\sum_{i=G_{1}+1}^{G_{2}} C_{i}+\sum_{i=1}^{x_{2}} C_{G_{2}}^{i}+\sum_{i=G_{2}+1}^{G_{3}} C_{i}+\sum_{i=1}^{x_{3}} C_{G_{3}}^{i}+\ldots \sum_{i=G_{n-1}+1}^{G_{n}} C_{i}+\sum_{i=1}^{x_{n}} C_{G_{n-1}}^{i}+\sum_{i=G_{n}+1}^{N-\left(G_{1}+G_{2}+\ldots+G_{n}\right)} C_{i}(1$

where $G_{1}, G_{2}, \ldots, G_{n-1}, G_{n}$ represent the customer that was serviced at the time the anticipatory customer's request comes in and $G_{1}+G_{2}+\cdots+G_{n}=N$.

So, the $D D$ for the third case from (10) and (12) is given by:

$$
D D=\frac{\sum_{i=1}^{x_{1}} c_{G_{1}}^{i}+\sum_{i=1}^{x_{2}} c_{G_{2}}^{i}+\ldots+\sum_{i=1}^{x_{n}} c_{G_{n}}^{i}}{\sum_{i=1}^{G_{1}} C_{i}+\sum_{i=1}^{x_{1}} c_{G_{1}}^{i}+\sum_{i=G_{1}+1}^{G_{2}} C_{i}+\sum_{i=1}^{x_{2}} c_{G_{2}}^{i}+\sum_{i=G_{2}+1}^{G_{3}} C_{i}+\sum_{i=1}^{x_{3}} c_{G_{3}}^{i}+\cdots+\sum_{i=G_{n-1}+1}^{G_{n}} C_{i}+\sum_{i=1}^{x_{n}} C_{G_{n-1}}^{i}+\sum_{i=G_{n}+1}^{N-\left(G_{1}+G_{2}+\ldots+G_{n}\right)} c_{i}}
$$

It is worthy of note that, in any of the three cases, the LRC cannot come first except at least one of the Early Request Customers have been attended to else such LRC becomes ERC.

The DD is a major dimension to classify DSVRP with stochastic requests. A moderate DD may be experienced for applications such as oil distribution, patient transports, or grocery delivery as in $[22,23]$. The scope of applications with high level DD comprises emergency vehicles or courier services as in $[24,25]$ and high DD can especially be found in emerging applications such as same-day delivery, demandresponsive transportation, or shared mobility [14, 26, 27]. For a detailed classification of DSVRP applications, see [28].

\subsection{Markov Decision Process (MDP) Model}

In here, the Dynamical situation in VRP has to be modeled. A model of the problem as a Markov Decision Process (MDP) was built and constructed [29]. The MDP will be split into three stages. The stages follow thus:

Stage 1: Compute the Pre-Decision State, $\mathrm{S}_{\mathrm{k}}$

The following notations are declared and used: Let the
Decision whenever the vehicle serves a customer be $k$ out of the total $N$. The Decision State, $S_{k}$, is given by the relation:

$$
S_{k}=\left(t_{k}, P_{k}, C_{k}, \theta_{k}, C_{k}^{\text {new }}\right)
$$

consisting of a Point in Time, $t_{k}$, Position of the Vehicle at the point in time, $\mathrm{P}_{\mathrm{k}}$. Set the number of customers not-yetserved in ERC as $C_{k}$. Assign to the tour plan sequence of LRC, (new requests), $\mathrm{C}_{\mathrm{k}}^{\text {new }}$ and let the Updated Planned Tour for ERC be given as:

$$
\theta_{\mathrm{k}}=\left(\mathrm{P}_{\mathrm{k}}, \mathrm{C}_{\mathrm{k}}^{1}, \mathrm{C}_{\mathrm{k}}^{2}, \mathrm{C}_{\mathrm{k}}^{3}, \ldots, \mathrm{C}_{\mathrm{k}}^{\mathrm{N}-\mathrm{x}}, \mathrm{C}_{0}\right)
$$

where $\mathrm{D}$ is the depot. Furthermore, the Decision State, $\mathrm{S}_{\mathrm{k}}$ contains the set of (l) new requests, $\mathrm{C}_{\mathrm{k}}^{\text {new }}$, defined by:

$$
\mathrm{C}_{\mathrm{k}}^{\text {new }}=\left\{\mathrm{C}_{\mathrm{k}}^{1}, \mathrm{C}_{\mathrm{k}}^{2}, \mathrm{C}_{\mathrm{k}}^{3}, \ldots, \mathrm{C}_{\mathrm{k}}^{\mathrm{l}}\right\}
$$

such that $l$ is a realization of a random variables that represents the number of new LRC request.

Stage 2: Compute the Decisions, $x$

Decisions, $x$, are made about the set of new LRCs to be served, $\mathrm{C}_{\mathrm{k}}^{\mathrm{x} \text {,assign }}$ of $\mathrm{C}_{\mathrm{k}}^{\text {new }}$, to assign leading to an updated set 
of customers to serve thus:

$$
\mathrm{C}_{\mathrm{k}}^{\mathrm{x}}=\mathrm{C}_{\mathrm{k}} \cup \mathrm{C}_{\mathrm{k}}^{\mathrm{x}, \text { assign }}
$$

A decision to further service the LRC calls for the update of the tour from $\theta_{\mathrm{k}}$ to $\theta_{\mathrm{k}}^{\mathrm{x}}$ following the sequences, $\mathrm{C}_{\mathrm{k}}^{\mathrm{x}}$ and determine the next customer to visit. A decision is feasible if the planned tour allows serving all customers and returning to the depot within the time limit. The immediate Reward of Decision, $x$, in State:

$$
S_{k}=\mathrm{R}\left(S_{k}, x\right)
$$

is the number of newly assigned LRC.

We break the decision transition between states into a decision transition and a post-decision state, Also, the stochastic transition from a post-decision state to a new decision state.

Stage 3: Compute the Post-Decision State $S_{k}^{x}$

The post decision state is of the form:

$$
\mathrm{S}_{\mathrm{k}}^{\mathrm{x}}=\left(\mathrm{t}_{\mathrm{k}}, \mathrm{C}_{\mathrm{k}}^{\mathrm{x}}, \theta_{\mathrm{k}}^{\mathrm{x}}\right)
$$

consisting of the point in time, $t_{k}$, the customers that are yet to be served, $\mathrm{C}_{\mathrm{k}}^{\mathrm{x}}$, and the plan tour, $\theta_{\mathrm{k}}^{\mathrm{x}}$, including the vehicle's position. The stochastic transition, $\omega_{k}$, results from the vehicle's distance travelled, service of the next customer, and a new (stochastic) set of LRCs. The transition updates the point of time, the vehicle position, the set of customers yet to be serve, and the set of newly arrived LRCs. The combination of decision and transition leads to a new decision state, $S_{k+1}$. The process terminates in state $S_{k}$ when the time limit is reached and the vehicle has returned to the depot.

\subsection{DSVRP Example}

The example below shows a situation whereby a vehicle leaves the depot and has to service some customers. Figure 1 presents an example of a Pre-decision state, a decision, and the resulting Post-decision state. On figure 1, the Green circles indicate the customers that have been serviced, the Red circles represent the point at which the vehicle is when new orders came in.

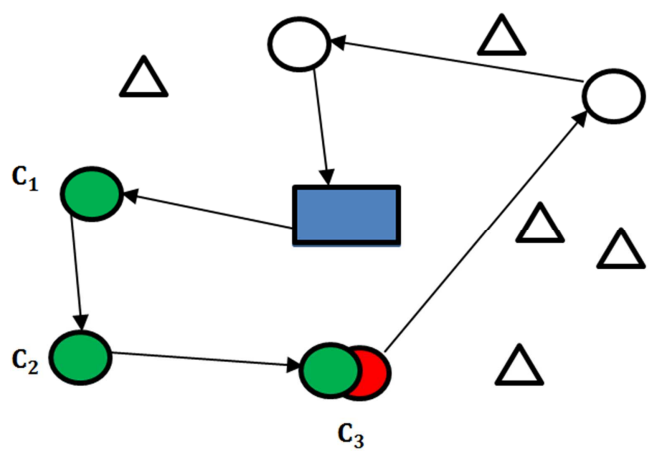

Figure 1. ERC Pre-Decision State.

The Triangles represent the LRC and the Rectangle represents the Depot. From (11), the Pre-State is given as:

$$
\mathrm{S}_{\mathrm{k}}=\left(540, \mathrm{P}_{\mathrm{k}},\left\{\mathrm{C}_{1}, \mathrm{C}_{2}, \mathrm{C}_{3}, \mathrm{C}_{4}, \mathrm{C}_{5}\right\},\left(\mathrm{P}_{\mathrm{k}}, \mathrm{C}_{\mathrm{k}}^{1}, \mathrm{C}_{\mathrm{k}}^{2}, \ldots, \mathrm{C}_{\mathrm{k}}^{\mathrm{N}-\mathrm{x}}, \mathrm{C}_{0}\right),\left\{\mathrm{C}_{\mathrm{k}}^{1}, \mathrm{C}_{\mathrm{k}}^{2}, \mathrm{C}_{\mathrm{k}}^{3}, \ldots, \mathrm{C}_{\mathrm{k}}^{\mathrm{l}}\right\}\right)
$$

The time, $t_{k}=540$ minutes, it is has depicted in figure 1 with Five customers: $\mathrm{C}_{1}, \mathrm{C}_{2}, \mathrm{C}_{3}, \mathrm{C}_{4}$ and $\mathrm{C}_{5}$, in succession following the direction of the arrow from the Depot, were assigned at the start of the tour. The vehicle has just serviced some customers and the current position of the vehicle which is a co-joined Green and Red Circles, meaning that, the customer has been serviced but the tour needs to be reoptimized due to LRC just coming in. The planned tour, $\theta_{\mathrm{k}}$, is indicated by the single arrows in figure 1 . The vehicle tour starts in $\mathrm{C}_{0}$ and serves the ERC in the order $\mathrm{C}_{1}, \mathrm{C}_{2}, \mathrm{C}_{3}, \mathrm{C}_{4}$ and $\mathrm{C}_{5}$ then, returns back to the depot, $\mathrm{C}_{0}$.

Five new LRC placed order requesting services after the tour has commenced thus:

$$
\mathrm{C}_{\mathrm{k}}^{\text {new }}=\left\{\mathrm{C}_{\mathrm{k}}^{1}, \mathrm{C}_{\mathrm{k}}^{2}, \mathrm{C}_{\mathrm{k}}^{3}, \mathrm{C}_{\mathrm{k}}^{4}, \mathrm{C}_{\mathrm{k}}^{5}\right\}
$$

Figure 2 below depicts the case in (9) in which the LRC comes between ERC just once which is the focus for now.

From Figure 2, $k=3$ since the request was placed after the third Green Dot. The Yellow Dot indicates the readiness to return to depot and the Red Rectangle indicates that the vehicle has returned to the depot. Then the LRC are given as:

$$
\mathrm{C}_{\mathrm{k}}^{\text {new }}=\mathrm{C}_{3}^{\text {new }}=\left\{\mathrm{C}_{3}^{1}, \mathrm{C}_{3}^{2}, \mathrm{C}_{3}^{3}, \mathrm{C}_{3}^{4}, \mathrm{C}_{3}^{5}\right\}
$$

Hence, the Decision

$$
x=\left(\mathrm{C}_{3}^{4}, \mathrm{C}_{3}^{5},\left(\mathrm{P}_{3}, \mathrm{C}_{5}, \mathrm{C}_{4}, \mathrm{C}_{3}^{3}, \mathrm{C}_{3}^{2}, \mathrm{C}_{3}^{1}, \mathrm{C}_{3}, \mathrm{C}_{2}, \mathrm{C}_{1}, \mathrm{C}_{0}\right)\right)
$$

determines the assignment of customer

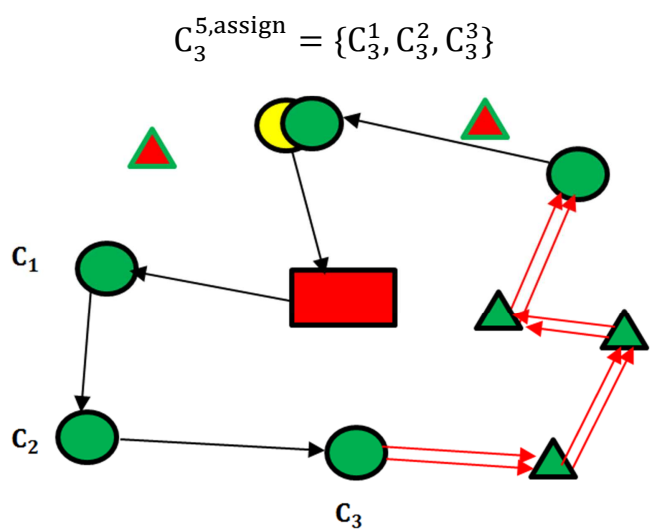

Figure 2. LRC Post-Decision State.

It must be noted that, while there were five LRC, only three could be serviced. This can be traced to the fact that; the vehicle has exhausted the carrying capacity or the due time to return to the depot has elapsed.

Then, updating the tour in (15) by considering the ERC and LRC's sequence gives:

$$
\theta_{3}^{5}=\left(\mathrm{P}_{3}, \mathrm{C}_{5}, \mathrm{C}_{4}, \mathrm{C}_{3}^{3}, \mathrm{C}_{3}^{2}, \mathrm{C}_{3}^{1} \mathrm{C}_{3}, \mathrm{C}_{2}, \mathrm{C}_{1}, \mathrm{C}_{0}\right)
$$

changing the previous sequence of servicing the customers to 
now include both the ERC and LRC.

The immediate reward which is the number of newly assigned LRC as in (18) gives rise to:

$$
\mathrm{R}\left(\mathrm{S}_{3}, 5\right)=3 .
$$

The application of the decisions, $x$, leads to a PostDecision State (19), thus:

$$
\mathrm{S}_{3}^{5}=\left(540,\left\{\mathrm{C}_{1}, \mathrm{C}_{2}, \mathrm{C}_{3}, \mathrm{C}_{4}, \mathrm{C}_{5}\right\}\left(\mathrm{P}_{3}, \mathrm{C}_{5}, \mathrm{C}_{4}, \mathrm{C}_{3}^{3}, \mathrm{C}_{3}^{2}, \mathrm{C}_{3}^{1}, \mathrm{C}_{3}, \mathrm{C}_{2}, \mathrm{C}_{1}, \mathrm{C}_{0}\right)\right)(27)
$$

The next locations to be serviced are $\mathrm{C}_{3}^{1}, \mathrm{C}_{3}^{2}$ and $\mathrm{C}_{3}^{3}$ indicated by the two arrows in Figure 2. Arriving at $C_{3}^{1}, C_{3}^{2}$ and $\mathrm{C}_{3}^{3}$, a set of new requests is revealed leading to the next state $S_{\mathrm{k}+1}$. In figure 2, an example of a dynamical situation in VRP is shown. In the example above, one un-capacitated vehicle has been used to service both advance and immediate request customers without any priorities placed on the LRC.

In a normal setting, the new customers should be included into the already planned routes without the order of the notyet-visited customers being changed and with minimal delay. This is the case depicted in figure 2. However, in real life situations, the inclusion of new customers will bring about a much more complicated task and will imply a re-planning of the part of the route system that has not been visited. This is illustrated by the figure 2 route where servicing of the new customers leads to a large detour in the whole system.

Generally, the more the restriction and complexity of the routing problem, the more complicated the inclusion of new dynamical customers will be. For instance, the inclusion of new customers in a time window constrained routing problem will usually be more difficult and tasking than in a non-time constrained problem. Note that in an online routing system, customers may even be denied service, if it is not possible to find a feasible spot to insert them.

Often, the policy of rejecting customers for the reason(s) of time horizon or vehicle carrying capacity, includes an order to serve the customers the following day or next trip or transferred to another vehicle. However, in some systems, like the pick-up of long-distance courier mail, the service provider or distributor will have to forward the customer to a competitor when they are not able to service them rather than blacklisting themselves.

\section{Problem Formulation}

Consider the VRP for a given time period, $\mathrm{T}$. Let $\mathrm{C}=\left\{c_{i}: i=0,1,2, \ldots, N\right\}$ be the set of $\mathrm{N}$ customers with $\mathrm{c}_{0}$ the depot. Let $V=\left\{v_{h} \mid h=1,2,3, \ldots, M\right\}$ be the set of $M$ homogenous vehicles stationed at the depot, $\mathrm{c}_{0}$. Every pair of locations $(i, j)$, where $i, j \leq N$ and $i \neq j$, is associated with a travel time, $t_{i j}$, from customer $c_{i}$ to $c_{j}$ and a distance traveled, $d(i, j)=d_{i j}$, that are symmetrical, i.e. $t_{i j}=t_{j i}$ and $d_{i j}=d_{j i}$ [30]. According to the authors, every customer, $c_{i}$, has the following basic requirements [32]:

CR1: A quantity, $\mathrm{q}_{\mathrm{i}}$, of product to be delivered by a vehicle, $\mathrm{V}_{\mathrm{h}}$.

CR2: The time, $t_{i j}$, required by the vehicle, $v_{h}$, to move from the depot or from a previous customer, $c_{i}$, to visit the next customer, $c_{j}$, to unload the quantity, $q_{i}$ and to leave for the next customer or return to the depot should all the customers in that route have been serviced or all the quantity exhausted.

CR3: The priority, $\delta_{i}$, of the customer, $c_{i}$, to be serviced by the vehicle, $\mathrm{v}_{\mathrm{h}}$.

The customers are serviced from only one depot with a homogeneous and limited fleet. The vehicles leave and ultimately return to the depot after the last customer has been serviced. There is a set $\mathrm{V}$ of vehicles with identical capacities. The capacity of each vehicle, $v_{h} \in V$ is represented by $Q_{h}$. Much as a customer has some requirements also the vehicle, $\mathrm{v}_{\mathrm{h}}$, have the following characteristics to be met thus:

VR1: A limited working period, $\mathrm{T}_{\mathrm{h}}$, of the vehicle, $\mathrm{v}_{\mathrm{h}}$, from the starting time, $\mathrm{T}_{\mathrm{h}}^{\mathrm{s}}$, to the finishing time, $\mathrm{T}_{\mathrm{h}}^{\mathrm{f}}$.

VR2: The fixed cost, $F C_{h}$, of wages to the driver and the loaders attached to each vehicle.

VR3: The carrying capacity, $Q_{h}$, of the vehicle.

On the customers' requirements and vehicles' characteristics, the following general assumptions are itemized [31]:

A1: The variable cost, $\mathrm{VC}_{\mathrm{ij}}$, is given as the cost of the least path from customer $c_{i}$ to customer $c_{j}$.

A2: The time, $t_{i j}$, is the corresponding travel time from customer $\mathrm{c}_{\mathrm{i}}$ to customer $\mathrm{c}_{\mathrm{j}}$.

A3: Let $R_{i}=\left\{r_{i}(1), \ldots, r_{i}(N)\right\}$ represent the set of routes for vehicle $v_{h}$, where $r_{i}(N)$ indicates the $n$th customer visited and $\mathrm{N}$ is the number of customers in the route. We also assume that every route terminates at the depot with $\mathrm{r}_{\mathrm{i}}(\mathrm{N}+$ 1) $=0$.

A4: The distance from where the vehicle can pack to unload to the warehouse or store of each customers is equal hence, the time to unload per item is fixed.

Let $\xi_{i j h}=1$ if vehicle $v_{h}$ visits customer $c_{j}$ immediately after visiting customer $\mathrm{c}_{\mathrm{i}}$ otherwise $\xi_{\mathrm{ijh}}=0$.

The VRP with Multiple Priorities is a multi-objective problem with the series, Min $\sum_{\mathrm{h}=1}^{4} \mathrm{~J}_{\mathrm{h}}$. Where Min $\mathrm{J}_{1}$ is meant to calculate the least path or distance carrying cost, $\mathrm{Min}_{2}$ is aimed at computing the fixed cost, $\mathrm{Min} \mathrm{J}_{3}$ is targeted at evaluating the priorities and $\mathrm{Min}_{4}$ is set at calculating the $\mathrm{OC}$ which is the sum of the ERC and LRC with:

$$
\begin{gathered}
\text { Min } \mathrm{J}_{1}=\alpha \sum_{i=1}^{N+x} \sum_{j=0}^{N+x}\left(\sum_{i=1}^{G} \mathrm{VC}_{\mathrm{i}}+\sum_{i=1}^{x} V C_{G}^{i}+\sum_{i=G+1}^{N-G} V C_{i}\right) \sum_{i=1}^{M} \xi_{\mathrm{ijh}} \\
\operatorname{Min~}_{2}=\beta \sum_{\mathrm{h}=1}^{\mathrm{M}}\left(F C_{\mathrm{h}} \sum_{\mathrm{j}=1}^{\mathrm{N}} \xi_{0 \mathrm{jh}}\right) \\
\operatorname{Min~J}_{3}=\gamma \sum_{\mathrm{j}=1}^{\mathrm{N}}\left(\delta_{\mathrm{j}} \sum_{\mathrm{i}=0}^{\mathrm{N}} \sum_{\mathrm{k}=1}^{\mathrm{M}} \xi_{\mathrm{ijh}}\right)
\end{gathered}
$$




$$
\operatorname{Min} \mathrm{J}_{4}=\sum_{i=1}^{N+x} \sum_{j=0}^{N+x}\left(\sum_{i=1}^{G} C_{i}+\sum_{i=1}^{x} C_{G}^{i}+\sum_{i=G+1}^{N-G} C_{i}\right) \sum_{i=1}^{M} \xi_{\mathrm{ijh}}
$$

where $\alpha, \beta$ and $\gamma$ are constants for weighting the terms corresponding to each objective [3].

On combining all the four sub objectives (28), (29), (30) and (31) we obtain:

$$
\begin{gathered}
\text { Min J }=\text { Min J }_{1}+\text { Min J }_{2}+\text { Min J }_{3}+\text { Min }_{4} \\
\text { Min J }=\alpha \sum_{i=1}^{N+x} \sum_{j=0}^{N+x}\left(\sum_{i=1}^{G} \mathrm{VC}_{\mathrm{i}}+\sum_{i=1}^{x} V C_{G}^{i}+\sum_{i=G+1}^{N-G} V C_{i}\right) \sum_{i=1}^{M} \xi_{\mathrm{ijh}}+\beta \sum_{\mathrm{h}=1}^{\mathrm{M}}\left(\mathrm{FC}_{\mathrm{k}} \sum_{\mathrm{j}=1}^{\mathrm{N}} \xi_{0 \mathrm{jh}}\right) \\
+\gamma \sum_{\mathrm{j}=1}^{\mathrm{N}}\left(\delta_{\mathrm{i}} \sum_{\mathrm{i}=0}^{\mathrm{N}} \sum_{\mathrm{h}=1}^{\mathrm{M}} \xi_{\mathrm{ijh}}\right)+\sum_{i=1}^{N+x} \sum_{j=0}^{N+x}\left(\sum_{i=1}^{G} C_{i}+\sum_{i=1}^{x} C_{G}^{i}+\sum_{i=G+1}^{N-G} C_{G+i}\right) \sum_{i=1}^{M} \xi_{\mathrm{ijh}}
\end{gathered}
$$

Subject to:

$$
\begin{gathered}
\sum_{\mathrm{i}=0}^{\mathrm{N}} \sum_{\mathrm{h}=1}^{\mathrm{M}} \xi_{\mathrm{ijh}} \leq 1, \\
\sum_{\mathrm{i}=0}^{\mathrm{N}} \xi_{\mathrm{iph}}-\sum_{\mathrm{j}=0}^{\mathrm{N}} \xi_{\mathrm{pjh}}=0, \mathrm{p}=0, \ldots, \mathrm{N} \\
\sum_{\mathrm{i}=1}^{\mathrm{N}}\left(\mathrm{q}_{\mathrm{i}} \sum_{\mathrm{j}=0}^{\mathrm{N}} \xi_{\mathrm{ijh}}\right) \leq \mathrm{FC}_{\mathrm{h}}, \mathrm{h}=1, \ldots, \\
\sum_{\mathrm{i}=0}^{\mathrm{N}} \sum_{\mathrm{j}=0}^{\mathrm{N}} \mathrm{t}_{\mathrm{ij}} \xi_{\mathrm{ijh}}+\sum_{\mathrm{i}=1}^{\mathrm{N}}\left(\mathrm{u}_{\mathrm{i}} \sum_{\mathrm{j}=0}^{\mathrm{N}} \xi_{\mathrm{ijh}}\right) \leq \mathrm{T}_{\mathrm{h}}^{\mathrm{f}}-\mathrm{T}_{\mathrm{h}}^{\mathrm{s}}, \\
\sum_{\mathrm{j}=1}^{\mathrm{N}} \xi_{0 \mathrm{jh}} \leq 1, \\
\mathrm{y}_{\mathrm{i}}-\mathrm{y}_{\mathrm{j}}+\mathrm{N} \sum_{\mathrm{k}=1}^{\mathrm{M}} \xi_{\mathrm{ijh}} \leq \mathrm{N}-1, \mathrm{i} \neq \mathrm{j} \\
\xi_{\mathrm{ijh}} \in\{0,1\} \forall \mathrm{i}, \mathrm{j}, \mathrm{h}
\end{gathered}
$$

The constraint (34) states that a customer can be visited at most once. Constraint (35) states that if a vehicle visits a customer, it must also depart from it. Constraint (36) is the capacity of the vehicle on each route. Constraint (37) is the working time limitations on each route. Constraint (38) states that a vehicle must be used at most once. Where $y_{i}$ is arbitrary, the relation (39) is the sub-tour-elimination condition derived for the Travelling Salesman Problem (TSP) and VRP as opined by Kohl, N., et al. (1999), and by JeanFrancois, C., et al, (2007), [32, 33, 1] respectively. This forces each route to pass through the depot. Constraint (40) is the integrality conditions.

If the aim of the VRP is to determine priority alone then, the first series, (28) and second series of (29) is set as zero while if the objective is to determine the priority as well as the cost alone then, the second series, (29) is set as zero. But where all the objectives are to be computed then, (33) holds.

\section{Recommendation}

For the success of the Dynamical situation in VRP, the timely, accurate and free flow of communication among the customer, dispatching center and the drivers of the vehicles is very paramount hence, the most up-to-date communication equipment are needed for the routing system. The equipment for determining the current position of the vehicles and the communication equipment for passing information on between the dispatching center (Depot) and the drivers in the vehicles will be discussed below.

Naturally, positioning equipment like the Global Positioning System (GPS) is essential to a dynamical situation in VRP. The GPS is a constellation of 24 satellites orbiting Earth that constantly send out signals, giving positions and time. Signals from three or four different satellites at any given time can provide receivers on the ground with enough information to calculate their precise location to within few meters depending on which version of the GPS system is put into use.

For well-structured routing system, the communication equipment between the vehicles and the dispatching center is essential. Mobile telephone communication systems are examples of technology capable of providing this information. Also, a dedicated radio base communication system could be used. The main difference in these technologies is the differences in initial and operating costs. While the mobile telephone communications system is relatively costly to operate, it has low initial costs because the basic technology is provided by the telephone companies and the GSM system today offers almost full coverage in most western industrialized countries, the initial costs in implementing a radio base communications system are on the high side, reason being that, the transmission masts will have to be put up and relatively expensive radio equipment must be installed in every vehicle. In all, a radio base communication system has very high initial costs, while the operating costs are almost negligible. Furthermore, the radio base system does not offer the same flexibility compared to the mobile telephone communications system.

It is expected that, the dispatching center knows which state is the vehicle and the driver at any given point in time. Alternatively, the driver sends a message about his current status and position to the dispatch center each time a customer has been serviced. However, as the above description indicates, this may prove to be infeasible for some applications due to the operating costs of this method but within a real-life setting the positioning information is transmitted at fixed intervals and an interpolation scheme is employed in order to estimate the positions of the vehicles.

Obviously, this approach does not offer the same level of information to the dispatcher to support the decision as to which vehicle to dispatch to the next waiting customer to be served. Should the latest information provided by the now idle driver/vehicle make the dispatcher change his mind on the current planned route, he will have to put a call through to the other drivers manually to update them of the changes in the current routes. The conclusion of this discussion must be that, a careful and meticulous analysis will have to show which approach to choose when designing the system. Of course, history has shown that, the price of communication has decreased rapidly in recent times. This could be a 
motivating factor for going for a telecommunication base system.

The technological advancements and improvements within Digital Strategy Roadmaps (DSR) and Geographical Information Systems (GIS) have been considerable in the last three decades. Most western industrialized countries now have detailed road network databases. The Road and Address Database offers a digital road database which is connected to detailed information on every address in the country that includes information on the zip codes, official street names, route numbers, road classification, major land topography, highest and lowest street number on both sides of the streets. In real-life application, it is vital that the solution algorithm chosen is capable of processing large amounts of geographical information fast enough to solve the problem online. However, issues related to computation of the shortest paths in a road network will not be treated in this paper. Although, these issues become necessary during implementation for online end user routing applications.

\section{Conclusion}

Dynamical situations in Vehicle Routing Problems is inevitable for we are dealing with real life situations that are characterized with change on daily bases. Such that no customer will be lost to close competitors rather, more customers would have been made hence, increasing the profit margin. Solving the class of problem becomes less difficult due to the advent and improvement in the information technology, the use of GSM, GPS and network facility that makes it possible else, it would have been a mirage and unattainable.

The vehicle must carry along with it an anticipatory quantity and give room for additional anticipatory time to cover supply and delivery time. There must be an information inter-connectivity between the customer, depot, the vehicle and other vehicles in the chain. When investigating dynamical situations in VRP systems, data generated randomly are used compared with real-life data. Reasons for this are: Firstly, the use of randomly generated data often enables more in-depth analyses, since the sets of data can be constructed such that other issues could be addressed alongside. Secondly, most real- life dynamical situations in VRP do not at the present capture all data needed for wholistic analyses of the routing problem. The geographical locations of all vehicles at the time new requests are received are one of the most commonly missing data items in the study of real-life problems.

\section{References}

[1] Jean-Francois, C., Gilbert, L., Martin W. P. S. and Daniele, V., (2007), Vehicle Routing, Handbook on OR and MS, Vol. 14, pg. 367-427.

[2] Christofides, N., Mingozzi, A. and Toth, P., (1976), Combinatorial Optimization, John Wiley \& Sons.

[3] Malkhi, D., Nisan, N., Pinkas, B. and Sella, Y., (2004),
Fairplay - a Secure Two-party Computation System, Proceedings of the thirteenth USENIX Security Symposium, pp. 287-302.

[4] Zaepfel, G. and Boegl, M., (2008), Multi-period Vehicle Routing and Crew Scheduling with Outsourcing Options. International Journal of Production Economics, 113 (2): 980996.

[5] Du, T., Wang, F. K., and Lu, P. Y., (2007), A Real-time Vehicle-dispatching System for Consolidating Milk Runs. Transportation Research Part E: Logistics and Transportation Review, 43 (5): 565-577.

[6] Gendreau, M., Guertin, F., Potvin, J. Y., and Seguin, R., (2006), Neighborhood Search Heuristics for a Dynamic Vehicle Dispatching Problem with Pickups and Deliveries. Transportation Research Part C: Emerging Technologies, 14 (3): $157-174$

[7] Larsen, A., (2000), The dynamic vehicle routing problem. Kgs. Lyngby, Denmark: Technical University of Denmark (DTU), IMM-Ph. D., No. 2000-73.

[8] Brotcorne, L., Laporte, G., and Semet, F., (2003), Ambulance Location and Relocation Models. European Journal of Operational Research, 147 (3): 451-463.

[9] Dantzig, G. and Ramser, J., (1959), The Truck Dispatching Problem, Management Science, 6 (1): 80-91.

[10] Hvattum, L. M., Lokketangen, A., Laporte, G., (2006), Solving a Dynamic and Stochastic Vehicle Routing Problem with a Sample Scenario Hedging Heuristic, Transportation Science, 40 (4): 421-438.

[11] Gendreau, M., Potvin, J.-Y., Braysy, O., Hasle, G., and Lokketangen, A., (2008), Metaheuristics for the Vehicle Routing Problem and its Extensions: Latest Advances and New Challenges, pages 143-169. Springer.

[12] Psaraftis, H. N., Wen, M. and Kontovas, C. A., (2016), Dynamic Vehicle Routing Problems: Three Decades and Counting. Networks, 67 (1): 3-31.

[13] Zhang, S., Ohlmann, J. W. and Thomas, B. W., (2018), Dynamic Orienteering on a Network of Queues, Transportation Science, 52 (3): 691-706.

[14] Goodson, J. C., Thomas, B. W. and Ohlmann, J. W., (2015), Restocking-based Rollout Policies for the Vehicle Routing Problem with Stochastic Demand and Duration limits. Transportation Science, 50 (2): 591-607.

[15] Schilde, M., Doerner, K. F. and Hart, R. F., (2014), Integrating Stochastic Time-dependent Travel Speed in Solution Methods for the Dynamic Dial-a-ride Problem. European Journal of Operational Research, 238 (1): 18-30.

[16] Speranza, M. G., (2018), Trends in Transportation and Logistics. European Journal of Operational Research, 264 (3): 830-836.

[17] Mitrovi'c-Mini'c, S., Krishnamurti, R., Laporte, G., (2004), Double Horizon-based Heuristics for the Dynamic Pickup and Delivery Problem with Time Windows. Transportation Research Part B, 38 (8): 669-685.

[18] Branke, J., Middendorf, M., Noeth, G., and Dessouky, M., (2005), Waiting Strategies for Dynamic Vehicle Routing. Transportation Science, 39 (3): 298-312. 
[19] Pureza, V., Laporte, G., (2008), Waiting and Buffering Strategies for the Dynamic Pickup and Delivery Problem with Time Windows. INFOR, 46 (3): 165-175.

[20] Jergensen, C., (1997), Driftsoptimering pa kraftvarmesystemer. $290 \mathrm{pp}$.

[21] Larsen, A., Madsen, O. B. G. and Solomon, M. M., (2002), Partially Dynamic Vehicle Routing-models and Algorithms. Journal of the Operational Research Society, 53 (6): 637-646.

[22] Ritzinger, U., Puchinger, J. and Hartl, R. F., (2014), Dynamic Programming Based Metaheuristics for the Dial-a-ride Problem. Annals of Operations Research, pages 1-18.

[23] Ehmke, J. F. and Campbell, A. M., (2014), Customer Acceptance Mechanisms for Home Deliveries in Metropolitan Areas. European Journal of Operational Research, 233 (1): 193-207.

[24] Maxwell, M. S., Restrepo, M., Henderson, S. G. and Topaloglu, H., (2010), Approximate dynamic programming for Ambulance Redeployment, INFORMS Journal on Computing, 22 (2): 266-38.

[25] Thomas, B. W., (2007), Waiting Strategies for Anticipating Service Requests from Known Customer Locations. Transportation Science, 41 (3): 319-331.

[26] Voccia, S. A., Campbell, A. M. and Thomas, B. W., (2018), The Same-day Delivery Problem for Online Purchases. Transportation Science.
[27] Brinkmann, J., Ulmer, M. W. and Mattfeld, D. C., (2015), Short-term Strategies for Stochastic Inventory Routing in Bike Sharing Systems. Transportation Research Procedia, 10: 364373.

[28] Ulmer, M. W., (2017), Approximate Dynamic Programming for Dynamic Vehicle Routing. Operations Research/Computer Science Interfaces Series. Springer.

[29] Puterman, M. L., (2014), Markov Decision Processes: Discrete Stochastic Dynamic Programming. John Wiley \& Sons, New York.

[30] Russell, R. A. and Chiang, W. C., (2006), Scatter Search for the Vehicle Routing Problem with Time Windows, European Journal of Operational Research, 169 (2): 606-622.

[31] Adebayo, K. J., Aderibigbe, F. M. and Dele-Rotimi, A. O., (2019) On Vehicle Routing Problems (VRP) with a Focus on Multiple Priorities. American Journal of Computational Mathematics, 9, 348-357.

[32] Miller, D. L., Pekny, J. F., (1995), A Staged Primal-dual Algorithm for Perfect B-matching with Edge Capacities. ORSA Journal on Computing 7, 298-320.

[33] Kohl, N., Desrosiers, J., Madsen, O. B. G., Solomon, M. M., and Soumis, F., (1999), 2-path Cuts for the Vehicle Routing Problem with Time Windows, Transportation Science 33: 101116. 